\title{
Obesity paradox in subarachnoid hemorrhage: a systematic review
}

\author{
Ilari Rautalin ${ }^{1,2}$ (D) Jaakko Kaprio ${ }^{2,3} \cdot$ Miikka Korja $^{1}$
}

Received: 15 May 2019 / Revised: 2 September 2019 / Accepted: 22 September 2019 / Published online: 29 October 2019

(C) The Author(s) 2019

\begin{abstract}
As the number of obese people is globally increasing, reports about the putative protective effect of obesity in life-threatening diseases, such as subarachnoid hemorrhage (SAH), are gaining more interest. This theory — the obesity paradox — is challenging to study, and the impact of obesity has remained unclear in survival of several critical illnesses, including SAH. Thus, we performed a systematic review to clarify the relation of obesity and SAH mortality. Our study protocol included systematic literature search in PubMed, Scopus, and Cochrane library databases, whereas risk-of-bias estimation and quality of each selected study were evaluated by the Critical Appraisal Skills Program and Cochrane Collaboration guidelines. A directional power analysis was performed to estimate sufficient sample size for significant results. From 176 reviewed studies, six fulfilled our eligibility criteria for qualitative analysis. One study found paradoxical effect (odds ratio, $\mathrm{OR}=0.83(0.74-0.92)$ ) between morbid obesity (body mass index (BMI) $>40$ ) and in-hospital SAH mortality, and another study found the effect between continuously increasing BMI and both short-term $(\mathrm{OR}=0.90(0.82-0.99))$ and long-term SAH mortalities $(\mathrm{OR}=0.92(0.85-0.98))$. However, according to our quality assessment, methodological shortcomings expose all reviewed studies to a high-risk-of-bias. Even though two studies suggest that obesity may protect SAH patients from death in the acute phase, all reviewed studies suffered from methodological shortcomings that have been typical in the research field of obesity paradox. Therefore, no definite conclusions could be drawn.
\end{abstract}

Keywords Obesity $\cdot$ Mortality $\cdot$ Subarachnoid hemorrhage $\cdot$ Systematic review

\section{Introduction}

Subarachnoid hemorrhage (SAH) is characterized as an acute cerebrovascular disease with multiple systemic complications and long intensive care unit (ICU) period [43]. Since roughly one-fourth [24] of SAH patients die suddenly before reaching a hospital ward and another one-fourth within the first 30 days [25], factors affecting the acute phase mortality are of interest.

Electronic supplementary material The online version of this article (https://doi.org/10.1007/s10143-019-01182-5) contains supplementary material, which is available to authorized users.

Ilari Rautalin

ilari.rautalin@helsinki.fi

1 Department of Neurosurgery, University of Helsinki and Helsinki University Hospital, P.O. Box 266, FI-00029 Helsinki, Finland

2 Department of Public Health, University of Helsinki, P.O. Box 20, FI-00014 Helsinki, Finland

3 Institute for Molecular Medicine FIMM, P.O. Box 20, FI-00014 Helsinki, Finland
Obesity is a well-known risk factor for increased morbidity and mortality in the general population $[13,14]$. As the number of obese persons is globally increasing [10], a theory called the obesity paradox has aroused increasing interest in recent years. According to this theory, overweight and obese people may have a survival benefit in various acute illnesses and medical conditions. In stroke subtypes other than SAH, such as intracerebral hemorrhage (ICH) [7, 21] and ischemic stroke $[20,22,35,45,47]$, being overweight or obese is associated with a favorable outcome. However, the true impact of BMI on the survival of any ICU patient group has remained unclear, and several studies have reported controversial results about the effects of obesity on the outcome of critically ill patients $[1,16,31]$. Since contributing comorbidities and a critical illness itself are presumably strong predictors of outcome, one of the major challenges in many previous obesity paradox studies has been the heterogeneity in the etiology of critical illnesses leading to ICU admission. Since only a few critical but relatively common acute illnesses among workingage and relatively healthy people invariably lead to long and expensive ICU periods that are further burdened with systemic complications, determining the role of obesity in survival has thus remained overly challenging. 
SAH, which affects working-age and relatively healthy people, is an optimal acute and critical illness to study the effect of obesity on short-term mortality. Contrary to other stroke subtypes, namely ICH and ischemic stroke [29], neither systematic reviews nor meta-analyses have been conducted on the topic of SAH survival and obesity. Therefore, our purpose was to review the previous literature on the possible effect of obesity on SAH survival. Because some studies have suggested that the obesity paradox can be explained by selection bias, confounding factors and inappropriate study designs [4, $9,12,19,41,42]$, we also tried to evaluate the quality of previous studies. Our hypothesis was that the obesity paradox is true (i.e., obesity (metabolic reservoir capacity) protects critically ill patients from death), and that this protective effect can be investigated by studying SAH patients, who often have lengthy and potentially catabolic ICU stays.

\section{Materials and methods}

Study protocol is available in the international prospective register of systematic reviews (PROSPERO, code CRD42018100003) and follows the checklist of Preferred Reporting Items for Systematic review and Meta-Analyses (PRISMA) [39] (Online Resource 1).

\section{Search strategy}

Details of the literature search strategy are described in Online Resource 2. Briefly, the search was based on both keywords and index terms and utilized three different databases: PubMed, Scopus, and Cochrane library. A study question and eligibility criteria were formulated using the four-step PICO (Patients, Intervention, Controls, Outcome) principle [17]. Case reports, case series, letters, commentaries, book chapters, animal studies, and descriptive studies without calculated risk estimates (hazard ratio (HR), odds ratio (OR) or relative risk (RR)) were excluded.

\section{Quality of studies}

To evaluate the heterogeneity and research methods of previous studies, we chose to use the Cochrane Collaboration Handbook and Critical Appraisal Skills Program (CASP) [15]. Based on the appropriate checklists, we focused on selection, detection, and measurement bias; limited and residual confounding; as well as on statistical power and causality between exposures and outcomes. To specify the sources of bias, we created six typical domains to describe these limitations wherein each domain had specific requirements classifying the studies into low, unclear or high-risk-of-bias categories (Online Resource 2). Finally, reviewed studies were grouped into either low-quality or high-quality categories.
To reach the high-quality category, all six domains had to be categorized as low-risk-of-bias.

\section{Statistical analyses}

A directional power analysis was performed to estimate sufficient sample size for significant results. The protective effects of being overweight, obese, and morbidly obese were optimistically estimated using an OR of 0.8 for BMI 25-29.9, OR $=0.7$ for BMI 30-39.9, and $\mathrm{OR}=0.6$ for $\mathrm{BMI} \geq 40$, with a reference 30 day mortality figure for the normal weight category $(\mathrm{BMI}=$ 18.5-24.9) approximated to $25 \%$ for hospital-based studies and $40 \%$ for studies that also included sudden-death SAHs before hospitalization [25]. Standard values were used for significance $(p<0.05)$ and statistical power $(p=0.8)$. Additionally, depending on the amount of high-quality studies, $I^{2}$ statistics for heterogeneity and random effects models for calculation of pooled estimates were prepared for possible meta-analysis.

\section{Results}

The literature search is depicted in Fig. 1. The search yielded 175 articles, and 1 article [36] was found through reference list screening of the 175 articles. Of the 176 publications, 5 [8, 11, $18,36,46]$ fulfilled the eligibility criteria. Additionally, one study [27] outside of our literature search fulfilled the criteria and thus was added to further qualitative analysis. All 177 articles were published in English.

Study characteristics varied considerably (Table 1). Three studies $[27,36,46]$ recruited cohorts and measured BMI values years before SAHs, whereas three $[8,11,18]$ were based on hospital admission registers with BMI values measured upon admission.

Study 1 In a Norwegian study, Sandvei et al. [36] prospectively examined incidence, case fatality, and preictal risk factors for survival among 214 (59\% male; mean age 47 years) SAH patients, including also sudden-death SAHs before hospitalization. Mean pre-SAH follow-up was 16 years, with postSAH follow-up divided into short-term (3 and 30 days) and long-term (6 months). BMI was measured years before $\mathrm{SAH}$ at enrollment and categorized according to WHO guidelines. No re-measurements of BMI were performed at admission.

Study 2 Yamada et al. [46] studied risk factors for fatal SAHs in a Japanese population-based prospective study. BMI calculations were based on self-administered questionnaires at enrollment, and BMI values were divided into low (BMI < 18.5), medium (BMI 18.5-24.9), and high (BMI $\geq 25$ ) categories. During the follow-up of 1,086,963 person-years and a median follow-up time of 9.9 years, 244 (36\% male; mean age 63 years) SAH deaths were reported. Mortality was verified 
Fig. 1 Flow chart of the literature search

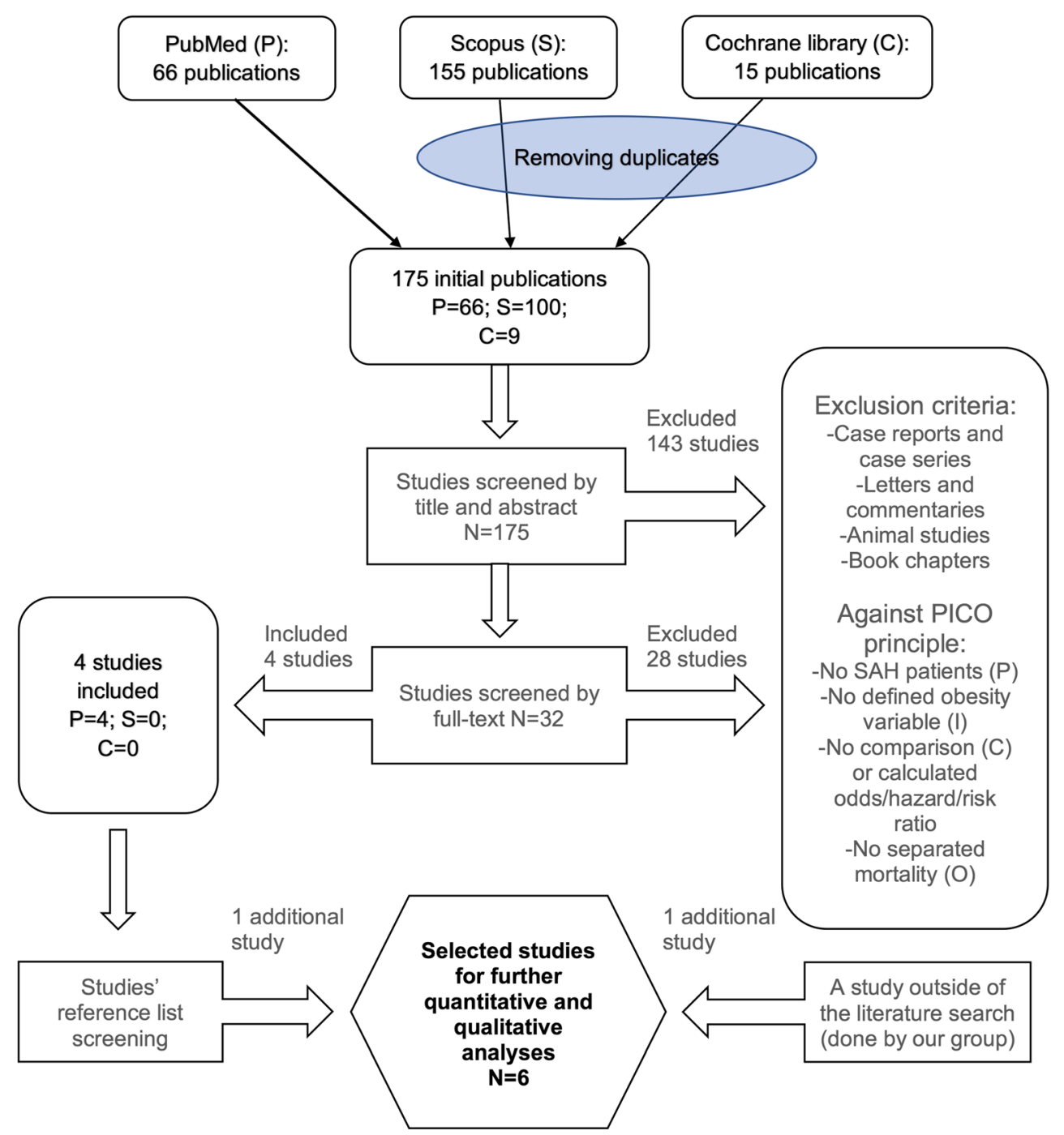

from the national cause of death register. No post-SAH follow-up was performed, and no re-measurements of BMI were performed on admission.

Study 3 In the third pre-SAH follow-up study, Lindbohm et al. [27] investigated risk factor differences between sudden-death and hospitalized SAH patients in a large prospective cohort study from Finland. During the follow-up of 1.52 million person-years and a median follow-up time of 23 years, 98 of 543 SAH patients (46\% male; mean age 61 years) died outside of hospital wards. BMI was measured years before SAH and analyzed only as a continuous variable. Neither hospital follow-up nor re-measurement of BMI was performed.

Study 4 Using the hospital-based register of the Nationwide Inpatient Sample (NIS) in the USA, Dasenbrock et al. [8] evaluated the association of both obesity (BMI 30-40) and morbid obesity (BMI > 40) with various outcomes after SAH. BMI categories were indirectly estimated by using registered ICD-9-CM diagnosis codes of obesity and morbid obesity, whereas controls consisted of NIS patients without either diagnosis. Between 2002 and 2011, 18,281 (31\% male; mean age 54 years) SAH patients were included in the analyses of in-hospital mortality.

Study 5 In another study based on the NIS, Elliott et al. [11] determined the impact of morbid obesity (BMI $\geq 40$ ) on non-traumatic SAH outcomes. In-hospital mortality analyses were based on 224,561 (40\% male; mean age 59 years) SAH patients diagnosed between years 2008 and 2013. BMI categories were also indirectly estimated by using registered ICD-9-CM codes of morbid obesity. NIS patients with $\mathrm{BMI}<40$ values served as a control group.

Study 6 In the third study from the USA, Hughes et al. [18] examined the association of obesity with both short-term (in-hospital or within 30 days after hospital discharge) and long-term (> 24 months) outcomes. BMI was measured at hospital admission and analyzed as a 
Table 1 Study characteristics

\begin{tabular}{|c|c|c|c|c|c|c|c|}
\hline First author & Country & Year & SAH cases & Mean age & Sex $(\%$ male $)$ & Follow-up time & Obesity categories \\
\hline \multicolumn{8}{|c|}{ Cohort studies with preictal follow-up } \\
\hline Sandvei & Norway & 2011 & 214 & 47.1 & 58.6 & $\begin{array}{l}\text { Before SAH: } 16 \text { years* } \\
\text { After SAH: } \\
6 \text { months }\end{array}$ & $\begin{array}{l}\text { BMI }<18.5 \\
\text { BMI 18.5-24.9 } \\
\text { BMI 25-29.9 } \\
\text { BMI } \geq 30\end{array}$ \\
\hline Yamada & Japan & 2003 & 244 & 63.1 & 36.1 & $\begin{array}{l}\text { Before SAH: } \\
9.9 \text { years* } \\
\text { After SAH: } \\
\text { NR }\end{array}$ & $\begin{array}{l}\text { BMI }<18.5 \\
\text { BMI } 18.5-24.9 \\
\text { BMI } \geq 25\end{array}$ \\
\hline Lindbohm & Finland & 2017 & 543 & 61.4 & 46.2 & $\begin{array}{l}\text { Before SAH:23 years* } \\
\text { After SAH: } \\
\text { Before hospitalization }\end{array}$ & Continuous BMI \\
\hline \multicolumn{8}{|c|}{ Cohort studies from hospital-based registers } \\
\hline Dasenbrock & USA & 2017 & 18,281 & 54.2 & 31.3 & In hospital & $\begin{array}{l}\text { NO }(\mathrm{BMI}<30) \\
\text { Obese }(\mathrm{BMI}=30-40) \\
\mathrm{MO}(\mathrm{BMI}>40)\end{array}$ \\
\hline Elliot & USA & 2017 & 224,561 & 59.4 & 40.2 & In hospital & $\begin{array}{l}\mathrm{NMO}(\mathrm{BMI} \leq 40) \\
\mathrm{MO}(\mathrm{BMI}>40)\end{array}$ \\
\hline Hughes & USA & 2015 & 305 & 55.8 & 35.2 & $\begin{array}{l}\text { Short-term (in hospital or } \\
30 \text { days after discharge) } \\
\text { Long-term ( } 24 \text { months) }\end{array}$ & $\begin{array}{l}\text { BMI }<25 \\
\text { BMI } 25-30 \\
\text { BMI } \geq 30\end{array}$ \\
\hline
\end{tabular}

$N R$ not reported, $M O$ morbidly obese patients, $N M O$ nonmorbidly obese patients, $N O$ nonobese patients

*Mean follow-up time

continuous variable in univariate and multivariate analyses. The hospital-based study included 305 (35\% male; mean age 56 years) SAH patients admitted between 2002 and 2011.

Risk-of-bias and quality of studies Based on the Cochrane Collaborator Handbook and CASP checklist, the selected domains for risk-of-bias estimation were the following: inclusion of sudden-death SAHs, valid obesity measurements, proper obesity analysis, control for confounding, comprehensive short-term follow-up, and sufficient sample size (Online Resource 2). None of the studies fulfilled the criteria for low risk-of-bias classification in all six domains; thus, all six studies $[8,11,18,27,36,46]$ were classified in the low-quality category (Table 2 ).
Power analysis Based on the pre-defined effect estimations and power analysis, we calculated that in studies with sudden-death SAHs (reference mortality 40\%), the minimum cohort size for significant results was $1128 \mathrm{SAH}$ patients (564 per category) when comparing overweight (BMI 25-30) and normal weight (BMI 18.5-24.9) patients (estimated OR = $0.8), 488$ (244 per category) when comparing obese (BMI > 30 ) and normal weight patients (estimated $\mathrm{OR}=0.7$ ) and 266 (133 per category) when comparing morbidly obese (BMI > 40) and normal weight patients (estimated OR $=0.6$ ). Additionally, in hospital-based studies (reference mortality $25 \%$ ), the minimum cohort sizes were 1094, 466, and 250, respectively. Based on these criteria, only two $[8,11]$ of the six studies consisted of an adequate sample size to reach sufficient statistical power (Table 2).

Table 2 Risk of bias evaluation. Plus sign (+) represents low-risk, NA (not applicable) unknown risk, and minus sign (-) high-risk-of-bias

\begin{tabular}{|c|c|c|c|c|c|c|}
\hline First author & Sudden-death SAHs & Obesity measurement & Obesity analysis & Short-term follow-up & Confounding control & Sufficient sample size \\
\hline \multicolumn{7}{|c|}{ Low-quality studies } \\
\hline Sandvei & + & - & + & + & - & - \\
\hline Yamada & NA & - & - & NA & + & - \\
\hline Lindbohm & + & - & - & - & + & - \\
\hline Dasenbrock & - & - & - & + & - & + \\
\hline Elliot & - & - & - & + & - & + \\
\hline Hughes & - & + & - & + & - & - \\
\hline
\end{tabular}


Short-term mortality The definition of "short term" varied between studies (Table 1). Only the Norwegian study [36] included sudden-death SAHs before hospitalization in their short-term (30-day) follow-up. However, contrary to Lindbohm et al. [27], no separate association analyses for outside hospital deaths were performed. Three $[8,11,18]$ studies, all conducted in the USA, started the follow-up from hospital admission. All these studies assessed in-hospital mortality rates and associations, but one study also included deaths until 30 days post-discharge.
Two studies $[11,18]$ found an association between BMI values and short-term SAH mortality (Table 3). First, Elliot et al. [11] found an inverse association (obesity paradox) between morbid obesity (BMI > 40) and SAH-related in-hospital mortality $(\mathrm{OR}=0.83)$ in a multivariate model (Table 3$)$. Second, Hughes et al. [18], with a somewhat longer follow-up of up to 30 days post-discharge, also found an inverse association (obesity paradox) between SAH mortality and increasing BMI values (Table 3 ) in both univariate (OR $=0.91)$ and multivariate $(\mathrm{OR}=0.90)$ models. The study reached statistical significance despite our power analysis

Table 3 SAH studies reporting associations between BMI and mortality

\begin{tabular}{|c|c|c|c|c|c|}
\hline \multirow[t]{2}{*}{ First author } & \multirow[t]{2}{*}{ Follow-up } & \multirow[t]{2}{*}{$\begin{array}{l}\text { Analyses } \\
\text { by sex }\end{array}$} & \multirow[t]{2}{*}{$\begin{array}{l}\text { BMI categories } \\
\left(\mathrm{kg} / \mathrm{m}^{2}\right)\end{array}$} & \multicolumn{2}{|l|}{$\begin{array}{l}\text { Risk of mortality } \\
\text { HR/OR }(95 \% \text { CI) }\end{array}$} \\
\hline & & & & Univariate analysis & Multivariate analysis \\
\hline \multicolumn{6}{|c|}{ Short-term mortality } \\
\hline \multirow[t]{8}{*}{ Sandvei } & \multirow[t]{4}{*}{3 days } & \multirow[t]{4}{*}{-} & Underweight $(<18.5)$ & - & - \\
\hline & & & Normal weight (18.5-24.9) & - & Reference \\
\hline & & & Overweight (25-29.9) & - & $0.6(0.3-1.4)$ \\
\hline & & & Obese $(\geq 30)$ & - & $1.1(0.4-3.1)$ \\
\hline & \multirow[t]{4}{*}{30 days } & \multirow[t]{4}{*}{-} & Underweight $(<18.5)$ & - & - \\
\hline & & & Normal weight (18.5-24.9) & - & Reference \\
\hline & & & Overweight (25-29.9) & - & $1.1(0.6-2.0)$ \\
\hline & & & Obese $(\geq 30)$ & - & $0.9(0.3-2.1)$ \\
\hline \multirow[t]{3}{*}{ Dasenbrock } & \multirow[t]{3}{*}{ In hospital } & \multirow[t]{3}{*}{-} & Non-obese $(<30)$ & Reference & Reference \\
\hline & & & Obese $(30-40)$ & $0.84(0.65-1.09)$ & $0.90(0.69-1.18)$ \\
\hline & & & Morbidly obese (> 40) & $0.75(0.54-1.06)$ & $0.77(0.54-1.11)$ \\
\hline \multirow[t]{2}{*}{ Elliot } & \multirow[t]{2}{*}{ In hospital } & \multirow[t]{2}{*}{-} & Nonmorbidly obese $(\leq 40)$ & - & Reference \\
\hline & & & Morbidly obese $(>40)$ & - & $0.83(0.74-0.92)$ \\
\hline Hughes & In hospital or 30 days after first discharge & - & Continuously & $0.91(0.84-0.98)$ & $0.90(0.82-0.99)$ \\
\hline \multicolumn{6}{|c|}{ Long-term mortality } \\
\hline \multirow[t]{4}{*}{ Sandvei } & \multirow[t]{4}{*}{6 months } & & Underweight $(<18.5)$ & - & $1.6(0.1-28.4)$ \\
\hline & & & Normal weight (18.5-24.9) & - & Reference \\
\hline & & & Overweight (25-29.9) & - & $1.0(0.5-1.9)$ \\
\hline & & & Obese $(\geq 30)$ & - & $1.0(0.4-2.4)$ \\
\hline Hughes & $>24$ months & - & Continuously & $0.92(0.86-0.97)$ & $0.92(0.85-0.98)$ \\
\hline \multicolumn{6}{|c|}{ No reported follow-up } \\
\hline \multirow[t]{9}{*}{ Yamada } & \multirow[t]{9}{*}{ NR } & \multirow[t]{3}{*}{ Both } & Low $(<18.5)$ & $1.63(1.06-2.50)$ & $1.82(0.98-3.38)$ \\
\hline & & & Moderate $(\geq 18.5$ and $<25.0)$ & Reference & Reference \\
\hline & & & $\operatorname{High}(\geq 25.0)$ & $1.02(0.73-1.43)$ & - \\
\hline & & \multirow[t]{3}{*}{ Men } & Low $(<18.5)$ & $1.85(0.91-3.75)$ & $2.72(1.03-7.23)$ \\
\hline & & & Moderate $(\geq 18.5$ and $<25.0)$ & Reference & Reference \\
\hline & & & $\operatorname{High}(\geq 25.0)$ & $0.73(0.38-1.38)$ & - \\
\hline & & \multirow[t]{3}{*}{ Women } & Low $(<18.5)$ & $1.52(0.89-2.60)$ & $1.44(0.64-3.21)$ \\
\hline & & & Moderate $(\geq 18.5$ and $<25.0)$ & Reference & Reference \\
\hline & & & $\operatorname{High}(\geq 25.0)$ & $1.16(0.79-1.73)$ & - \\
\hline Lindbohm & Before hospitalization & - & Continuously & - & $0.86(0.68-1.09)$ \\
\hline
\end{tabular}

$B M I$ body mass index, $C I$ confidence interval, $H R$ hazard ratio, NR not reported, $O R$ odds ratio 
suggested that the cohort size was inadequate to reach significance. Two other studies $[8,36]$ concluded that no significant associations existed between BMI and short-term SAH mortality. The last two studies $[27,46]$ did not define the time frame of short-term follow-up.

Long-term mortality In addition to short-term mortality, two studies also analyzed long-term mortality (Table 3). Only Hughes et al. [18] found an inverse association (obesity paradox) between continuously increasing BMI values and longterm (> 24 months) post-SAH mortality in both univariate $(\mathrm{OR}=0.92)$ and multivariate $(\mathrm{OR}=0.92)$ models (Table 3$)$.

Mortality without a defined time frame of follow-up Two studies [27, 46] did not define a follow-up time for hospitalized patients. First, Yamada et al. [46] found an association between underweight $(\mathrm{BMI}<18.5)$ patients and fatal SAHs in general (Table 3). In a univariate analysis, the association was reported for both sexes $(\mathrm{HR}=1.63)$, whereas in the multivariate analysis, low BMI remained an independent risk factor only for men $(H R=2.73)$ (Table 3$)$. The study found no significant associations between overweight/obesity $(\mathrm{BMI} \geq 25)$ and mortality when using normal weight patients as a reference group (Table 3). Second, Lindbohm et al. [27] reported an inverse association between increasing BMI and all SAH cases (HR $=0.90$ ). However, when analyses were done separately for suddenly died and hospitalized SAH patients, no significance was reached. Additionally, in comparison analysis, no BMI difference was found between sudden-death and hospitalized SAH patients ( $p=0.81$ for men, $p=0.76$ for women).

Meta-analysis Due to methodological differences and the absence of high-quality studies, a quantitative meta-analysis was impractical to conduct.

\section{Discussion}

Two studies $[11,18]$ found an obesity paradox in SAH mortality, but methodological shortcomings relegate these studies into a high-risk-of-bias category; therefore, firm conclusions about a protective effect of obesity in SAH mortality cannot be drawn. Although the reported obesity paradox can be explained at least partly by methodological shortcomings, physiological mechanisms explaining better survival of obese patients are also possible. For example, protective mechanisms might relate to an increased metabolic reserve of adipose or muscle mass, which could improve tolerance of major catabolic and inflammatory events among critically ill patients treated in ICUs. Supporting this theory, a few studies [3, 8, 34] have related obesity to more favorable outcomes, measured using the modified Rankin Scale (mRS), and milder overweight to lower complication rates as well. On the other hand, other studies have reported contradictory findings [33, 37, 44].

While a protective effect of obesity in SAH survival is still questionable, morbidly obese SAH patients in particular seem to have several disadvantages. In SAH, postoperative infections [8], venous thromboembolisms $[8,26,38]$, acute respiratory failure [11], fever [32], prolonged hospitalization [11], and non-routine discharge [8] are associated with higher BMI. Since obese patients tend to have higher complication rates and longer hospital stays, studies on SAH outcome may have a performance bias relating to differing short-term treatments. In addition to higher complication rates, morbid obesity increases hospital costs in SAH [11]. However, if the obesity paradox is true, the higher treatment costs and longer hospitalizations of obese or morbidly obese patients could be better justified.

In other stroke subtypes, sudden deaths before hospitalization are less common than in SAH $[5,40]$. In SAH, nearly half of 1-year deaths occur before hospitalization and around $90 \%$ within the first 30 days [25]. Since hospitalized SAH patients often stay 2-3 weeks in the ICU and the majority of deaths occur within the first month following the ictus, a follow-up time of 30 days seems optimal in assessing any effects of obesity on mortality in SAH. Moreover, since less than $5 \%$ of SAH patients die between 30 days and 1 year [25], SAH appears to be a rather optimal acute disease to examine any effects of obesity on the short-term survival of critically ill patients.

\section{Limitations in previous studies}

Four studies [8, 11, 18, 46] excluded sudden-death SAHs. Studies missing roughly $25 \%$ of all SAHs and about $50 \%$ of fatal cases have a theoretical risk for selection bias. Even though one previous study did not find BMI differences between sudden-death and hospitalized SAH patients [27], no comparison was made between fatal cases only. Since the same study found that sudden-death SAH patients have significantly worse overall risk factor profiles than the hospitalized patients [27], even a minimal BMI difference between sudden SAH deaths and hospitalized fatal SAHs may distort hospital-based registers to the extent of losing study reliability. In the Norwegian study with both sudden and hospitalized SAH deaths in the analyses [36], no comparisons between the groups were made.

With regard to obesity assessments, all six studies had several shortcomings. First, three studies $[27,36,46]$ measured BMI only at enrollment. Since most adults gain weight until serious illnesses late in life or until the onset of sarcopenia [28], those who were overweight at enrollment are likely to also be overweight or obese at the onset of SAH. Therefore, the use of premorbid BMI is likely underestimating the effect of obesity. To avoid false negative results, future studies with long preictal monitoring periods could perform BMI remeasurements at admission. Second, two studies analyzed 
obesity as a continuous variable [18, 27], while three used only combined BMI categories, such as non-obese (BMI < 30) [8], non-morbidly obese $(\mathrm{BMI}<40)$ [11], or high BMI $(\mathrm{BMI}>25)$ [46] categories. Therefore, statistical analyses of weight extremes could not be conducted. Since the effect of obesity is unlikely linear (i.e., the fatter the better) and differences by obese severity have also been reported in SAH mortality, the comparison between overweight (BMI 25-29.9), obese (BMI 30-39.9), and morbidly obese (BMI $\geq 40$ ) SAH patients is difficult to conduct if, for example, all patients with BMI values over 25 are combined into one category. Third, two studies estimated BMIs by relying on physician reports $[8,11]$, and one study used self-administered questionnaires [46] without controlling for measurement bias. All studies used only BMI as an obesity variable. Since BMI sensitivity has been reported to be only $50 \%$ for high mass of body fat [30], future studies could also consider other obesity variables with better sensitivity such as waist and hip circumference measurements, magnetic resonance imaging (MRI), dualenergy X-ray absorptiometry (DEXA), or bio-impedance of body.

Only two studies, NIS register-based studies $[8,11]$ from the USA, fulfilled the criteria of sufficient sample size providing high statistical power for analyses. Any protective effect of high BMI values is likely smaller than our optimistic estimation, so the other four studies $[18,27,36,46]$ likely had insufficient power to achieve significant results. In addition, our directional analysis did not consider effects of potential confounding, thus further underestimating the required cohort sizes.

\section{Confounders in studies on the obesity paradox in SAH}

In a recent large prospective follow-up study, Lindbohm et al. [27] found that the risk for sudden SAH death seems to be highest among people with the worst risk factor profile (i.e., among heavy smokers and people with high blood pressure values). Both smoking [6] and high blood pressure values [14] have been associated with obesity. In terms of smoking, a large study of nearly 300,000 participants [19] reported that the observed obesity paradox in patients with cardiovascular diseases vanished after analyzing only non-smokers. For these reasons, we presumed that both smoking and blood pressure should be treated as confounders in low-risk-of-bias studies of the obesity paradox in SAH. Overall, two studies [27, 46] fulfilled this criterion (Tables 2 and 3 ).

As potential other confounders, obese patients often suffer from metabolic syndrome, diabetes, coronary artery disease, and peripheral vascular disease. Although these comorbidities might also impact the survival of critically ill patients, no high-quality evidence exists, suggesting an association between these comorbidities and short-term SAH mortality. Moreover, as the prevalence of these comorbidities is low in
SAH patients $[2,23]$, we did not consider these factors as likely confounders, and therefore, they were not regarded mandatory in statistical analyses to reach the classification of low-risk-of-bias. In contrast, other factors, such as SAH severity and rebleeding, have been associated with higher mortality rates; no evidence relates these findings to obesity groups. Furthermore, Rinaldo et al. [34] found an effect of BMI on SAH outcome, and this effect varied depending on the treatment modality (clipping versus coiling). However, the study found no differences after using the validated WHO categories for BMI. In addition, the characteristics between treatment groups varied significantly. Since convincing evidence of treatment confounding in SAH does not exist, we did not include this factor in bias classifications. In brief, future studies about obesity paradox in SAH should try to consider at least smoking and hypertension as potential confounders and perhaps evaluate if composite variables of cardiovascular risk modify the relation of BMI and SAH mortality.

Although the literature search was done systematically utilizing three different databases, we may have missed some relevant publications. For example, large studies with comprehensive and wide-ranging risk factor assessments may have neglected minor and especially negative findings in their indexing. This type of reporting and indexing bias may cause the exclusion of studies like the one by Lindbohm et al. [27]. Furthermore, we used only English search terms, assuming that clinically relevant studies have been conducted in large hospitals and subsequently reported in English. Lastly, we were not able to conduct a meta-analysis due to methodological differences and the absence of high-quality studies.

\section{Conclusions}

None of the reviewed obesity paradox studies were confirmative, and effects of obesity on the risk of death among critically ill SAH patients remain to be studied in more detail. However, since the poor-quality evidence suggests that the obesity paradox may exist in SAH and since the number of obese patients is globally increasing, future studies on the topic are encouraged. To avoid major biases, future studies should perhaps try to include sudden-death SAHs in their analyses, use valid and timely obesity measurements on all SAH patients, and aim for reasonable cohort sizes.

Acknowledgements We would like to thank information specialist Tiina Heino for consultation and additional advice on the search protocol and Alyce Whipp for language revision.

Funding information Open access funding provided by University of Helsinki including Helsinki University Hospital. IR received research grants from the Finnish Medical Foundation, Juho Vainio Foundation, Maire Taponen Foundation, and Petri Honkanen Foundation. 
Compliance with ethical standards Study protocol is available in the international prospective register of systematic reviews (PROSPERO, code CRD42018100003) and follows the checklist of Preferred Reporting Items for Systematic review and Meta-Analyses (PRISMA).

Disclaimer Reported foundations have no personal or institutional financial interests concerning this study.

Conflict of interest The authors declare that they have no conflict of interest.

Ethical approval This article does not contain any studies with human participants or animals performed by any of the authors.

Informed consent The review was based on systematic literature search and thus, included no personal data.

Open Access This article is distributed under the terms of the Creative Commons Attribution 4.0 International License (http:// creativecommons.org/licenses/by/4.0/), which permits unrestricted use, distribution, and reproduction in any medium, provided you give appropriate credit to the original author(s) and the source, provide a link to the Creative Commons license, and indicate if changes were made.

\section{References}

1. Abhyankar S, Leishear K, Callaghan FM, Demner-Fushman D, McDonald CJ (2012) Lower short- and long-term mortality associated with overweight and obesity in a large cohort study of adult intensive care unit patients. Crit Care 16:R235. https://doi.org/10. 1186/cc11903

2. Adams HP Jr, Putman SF, Kassell NF, Torner JC (1984) Prevalence of diabetes mellitus among patients with subarachnoid hemorrhage. Arch Neurol 41:1033-1035. https://doi.org/10.1001/archneur. 1984.04050210031009

3. Badjatia N, Monahan A, Carpenter A, Zimmerman J, Schmidt JM, Claassen J, Connolly ES, Mayer SA, Karmally W, Seres D (2015) Inflammation, negative nitrogen balance, and outcome after aneurysmal subarachnoid hemorrhage. Neurology 84:680-687. https:// doi.org/10.1212/WNL.0000000000001259

4. Banack HR, Kaufman JS (2013) The "obesity paradox" explained. Epidemiology 24:461-462. https://doi.org/10.1097/EDE. 0b013e31828c776c

5. Black M, Graham DI (2002) Sudden unexplained death in adults caused by intracranial pathology. J Clin Pathol 55:44-50

6. Carreras-Torres R, Johansson M, Haycock PC, Relton CL, Davey Smith G, Brennan P, Martin RM (2018) Role of obesity in smoking behaviour: Mendelian randomisation study in UK Biobank. BMJ 361:k1767. https://doi.org/10.1136/bmj.k1767

7. Dangayach NS, Grewal HS, De Marchis GM, Sefcik RK, Bruce R, Chhatlani A, Connolly ES, Falo MC, Agarwal S, Claassen J, Schmidt JM, Mayer SA (2018) Does the obesity paradox predict functional outcome in intracerebral hemorrhage? J Neurosurg 129: 1125-1129. https://doi.org/10.3171/2017.5.JNS163266

8. Dasenbrock HH, Nguyen MO, Frerichs KU, Guttieres D, Gormley WB, Ali Aziz-Sultan M, Du R (2017) The impact of body habitus on outcomes after aneurysmal subarachnoid hemorrhage: a Nationwide Inpatient Sample analysis. J Neurosurg 127:36-46. https://doi.org/10.3171/2016.4.JNS152562
9. Dehlendorff C, Andersen KK, Olsen TS (2014) Body mass index and death by stroke: no obesity paradox. JAMA Neurology 71: 978-984. https://doi.org/10.1001/jamaneurol.2014.1017

10. Eckel RH, York DA, Rossner S, Hubbard V, Caterson I, St Jeor ST, Hayman LL, Mullis RM, Blair SN, American Heart A (2004) Prevention Conference VII: obesity, a worldwide epidemic related to heart disease and stroke: executive summary. Circulation 110: 2968-2975. https://doi.org/10.1161/01.CIR.0000140086.88453. $9 \mathrm{~A}$

11. Elliott RS, Godoy DA, Michalek JE, Behrouz R, Elsehety MA, Hafeez S, Rios D, Seifi A (2017) The effect of morbid obesity on subarachnoid hemorrhage prognosis in the United States. World Neurosurg 105:732-736. https://doi.org/10.1016/j.wneu.2017.06. 068

12. Ferreira I, Stehouwer CD (2012) Obesity paradox or inappropriate study designs? Time for life-course epidemiology. J Hypertens 30: 2271-2275. https://doi.org/10.1097/HJH.0b013e32835b4fe0

13. Flegal KM, Kit BK, Orpana H, Graubard BI (2013) Association of all-cause mortality with overweight and obesity using standard body mass index categories: a systematic review and meta-analysis. JAMA 309:71-82. https://doi.org/10.1001/jama.2012.113905

14. Guh DP, Zhang W, Bansback N, Amarsi Z, Birmingham CL, Anis AH (2009) The incidence of co-morbidities related to obesity and overweight: a systematic review and meta-analysis. BMC Public Health 9:88. https://doi.org/10.1186/1471-2458-9-88

15. Harrison JK, Reid J, Quinn TJ, Shenkin SD (2017) Using quality assessment tools to critically appraise ageing research: a guide for clinicians. Age Ageing 46:359-365. https://doi.org/10.1093/ ageing/afw223

16. Hogue CW Jr, Stearns JD, Colantuoni E, Robinson KA, Stierer T, Mitter N, Pronovost PJ, Needham DM (2009) The impact of obesity on outcomes after critical illness: a meta-analysis. Intensive Care Med 35:1152-1170. https://doi.org/10.1007/s00134-009$1424-5$

17. Huang X, Lin J, Demner-Fushman D (2006) Evaluation of PICO as a knowledge representation for clinical questions. AMIA Annu Symp Proc 2006:359-363

18. Hughes JD, Samarage M, Burrows AM, Lanzino G, Rabinstein AA (2015) Body mass index and aneurysmal subarachnoid hemorrhage: decreasing mortality with increasing body mass index. World Neurosurg 84:1598-1604. https://doi.org/10.1016/j.wneu. 2015.07.019

19. Iliodromiti S, Celis-Morales CA, Lyall DM, Anderson J, Gray SR, Mackay DF, Nelson SM, Welsh P, Pell JP, Gill JMR, Sattar N (2018) The impact of confounding on the associations of different adiposity measures with the incidence of cardiovascular disease: a cohort study of 296535 adults of white European descent. Eur Heart J 39:1514-1520. https://doi.org/10.1093/eurheartj/ehy057

20. Kim BJ, Lee SH, Jung KH, Yu KH, Lee BC, Roh JK, For Korean Stroke Registry i (2012) Dynamics of obesity paradox after stroke, related to time from onset, age, and causes of death. Neurology 79: 856-863. https://doi.org/10.1212/WNL.0b013e318266fad1

21. Kim BJ, Lee SH, Ryu WS, Kim CK, Lee J, Yoon BW (2011) Paradoxical longevity in obese patients with intracerebral hemorrhage. Neurology 76:567-573. https://doi.org/10.1212/WNL. 0b013e31820b7667

22. Kim Y, Kim CK, Jung S, Yoon BW, Lee SH (2015) Obesity-stroke paradox and initial neurological severity. J Neurol Neurosurg Psychiatry 86:743-747. https://doi.org/10.1136/jnnp-2014-308664

23. Knekt P, Reunanen A, Aho K, Heliovaara M, Rissanen A, Aromaa A, Impivaara O (1991) Risk factors for subarachnoid hemorrhage in a longitudinal population study. J Clin Epidemiol 44:933-939

24. Korja M, Lehto H, Juvela S, Kaprio J (2016) Incidence of subarachnoid hemorrhage is decreasing together with decreasing smoking rates. Neurology 87:1118-1123. https://doi.org/10.1212/WNL. 0000000000003091 
25. Korja M, Silventoinen K, Laatikainen T, Jousilahti P, Salomaa V, Kaprio J (2013) Cause-specific mortality of 1-year survivors of subarachnoid hemorrhage. Neurology 80:481-486. https://doi.org/ 10.1212/WNL.0b013e31827f0fb5

26. Kshettry VR, Rosenbaum BP, Seicean A, Kelly ML, Schiltz NK, Weil RJ (2014) Incidence and risk factors associated with inhospital venous thromboembolism after aneurysmal subarachnoid hemorrhage. J Clin Neurosci 21:282-286. https://doi.org/10.1016/j. jocn.2013.07.003

27. Lindbohm JV, Kaprio J, Jousilahti P, Salomaa V, Korja M (2017) Risk factors of sudden death from subarachnoid hemorrhage. Stroke 48:2399-2404. https://doi.org/10.1161/STROKEAHA.117. 018118

28. Ng M, Fleming T, Robinson M, Thomson B, Graetz N, Margono C, Mullany EC, Biryukov S, Abbafati C, Abera SF, Abraham JP, AbuRmeileh NM, Achoki T, AlBuhairan FS, Alemu ZA, Alfonso R, Ali MK, Ali R, Guzman NA, Ammar W, Anwari P, Banerjee A, Barquera S, Basu S, Bennett DA, Bhutta Z, Blore J, Cabral N, Nonato IC, Chang JC, Chowdhury R, Courville KJ, Criqui MH, Cundiff DK, Dabhadkar KC, Dandona L, Davis A, Dayama A, Dharmaratne SD, Ding EL, Durrani AM, Esteghamati A, Farzadfar F, Fay DF, Feigin VL, Flaxman A, Forouzanfar MH, Goto A, Green MA, Gupta R, Hafezi-Nejad N, Hankey GJ, Harewood HC, Havmoeller R, Hay S, Hernandez L, Husseini A, Idrisov BT, Ikeda N, Islami F, Jahangir E, Jassal SK, Jee SH, Jeffreys M, Jonas JB, Kabagambe EK, Khalifa SE, Kengne AP, Khader YS, Khang YH, Kim D, Kimokoti RW, Kinge JM, Kokubo Y, Kosen S, Kwan G, Lai T, Leinsalu M, Li Y, Liang X, Liu S, Logroscino G, Lotufo PA, Lu Y, Ma J, Mainoo NK, Mensah GA, Merriman TR, Mokdad AH, Moschandreas J, Naghavi M, Naheed A, Nand D, Narayan KM, Nelson EL, Neuhouser ML, Nisar MI, Ohkubo T, Oti SO, Pedroza A, Prabhakaran D, Roy N, Sampson U, Seo H, Sepanlou SG, Shibuya K, Shiri R, Shiue I, Singh GM, Singh JA, Skirbekk V, Stapelberg NJ, Sturua L, Sykes BL, Tobias M, Tran BX, Trasande L, Toyoshima H, van de Vijver S, Vasankari TJ, Veerman JL, Velasquez-Melendez G, Vlassov VV, Vollset SE, Vos T, Wang C, Wang X, Weiderpass E, Werdecker A, Wright JL, Yang YC, Yatsuya H, Yoon J, Yoon SJ, Zhao Y, Zhou M, Zhu S, Lopez AD, Murray CJ, Gakidou E (2014) Global, regional, and national prevalence of overweight and obesity in children and adults during 1980-2013: a systematic analysis for the Global Burden of Disease Study 2013. Lancet 384:766-781. https://doi.org/10.1016/S0140-6736(14)60460-8

29. Oesch L, Tatlisumak T, Arnold M, Sarikaya H (2017) Obesity paradox in stroke - myth or reality? A systematic review. PLoS One 12: e0171334. https://doi.org/10.1371/journal.pone.0171334

30. Okorodudu DO, Jumean MF, Montori VM, Romero-Corral A, Somers VK, Erwin PJ, Lopez-Jimenez F (2010) Diagnostic performance of body mass index to identify obesity as defined by body adiposity: a systematic review and meta-analysis. Int J Obes 34 : 791-799. https://doi.org/10.1038/ijo.2010.5

31. Oliveros H, Villamor E (2008) Obesity and mortality in critically ill adults: a systematic review and meta-analysis. Obesity (Silver Spring) 16:515-521. https://doi.org/10.1038/oby.2007.102

32. Park YK, Yi HJ, Choi KS, Lee YJ, Chun HJ, Kwon SM (2018) Predictive factors of fever after aneurysmal subarachnoid hemorrhage and its impact on delayed cerebral ischemia and clinical outcomes. World Neurosurg. https://doi.org/10.1016/j.wneu.2018.03. 030

33. Platz J, Guresir E, Schuss P, Konczalla J, Seifert V, Vatter H (2013) The impact of the body mass index on outcome after subarachnoid hemorrhage: is there an obesity paradox in SAH? A retrospective analysis. Neurosurgery 73:201-208. https://doi.org/10.1227/01. neu.0000430322.17000.82

34. Rinaldo L, Hughes JD, Rabinstein AA, Lanzino G (2018) Effect of body mass index on outcome after aneurysmal subarachnoid hemorrhage treated with clipping versus coiling. J Neurosurg 129: 658-669. https://doi.org/10.3171/2017.4.JNS17557

35. Ryu WS, Lee SH, Kim CK, Kim BJ, Yoon BW (2011) Body mass index, initial neurological severity and long-term mortality in ischemic stroke. Cerebrovasc Dis 32:170-176. https://doi.org/10.1159/ 000328250

36. Sandvei MS, Mathiesen EB, Vatten LJ, Muller TB, Lindekleiv H, Ingebrigtsen T, Njolstad I, Wilsgaard T, Lochen ML, Vik A, Romundstad PR (2011) Incidence and mortality of aneurysmal subarachnoid hemorrhage in two Norwegian cohorts, 1984-2007. Neurology 77:1833-1839. https://doi.org/10.1212/WNL. 0b013e3182377de3

37. Schultheiss KE, Jang YG, Yanowitch RN, Tolentino J, Curry DJ, Luders J, Asgarzadie-Gadim F, Macdonald RL (2009) Fat and neurosurgery: does obesity affect outcome after intracranial surgery? Neurosurgery 64:316-326; discussion 326-317. https://doi.org/10. 1227/01.NEU.0000336329.90648.17

38. Serrone JC, Wash EM, Hartings JA, Andaluz N, Zuccarello M (2013) Venous thromboembolism in subarachnoid hemorrhage. World Neurosurg 80:859-863. https://doi.org/10.1016/j.wneu. 2013.01.012

39. Shamseer L, Moher D, Clarke M, Ghersi D, Liberati A, Petticrew M, Shekelle P, Stewart LA, Group P-P (2015) Preferred reporting items for systematic review and meta-analysis protocols (PRISMAP) 2015: elaboration and explanation. BMJ 350:g7647. https://doi. org/10.1136/bmj.g7647

40. Soros P, Hachinski V (2012) Cardiovascular and neurological causes of sudden death after ischaemic stroke. Lancet Neurol 11: 179-188. https://doi.org/10.1016/S1474-4422(11)70291-5

41. Staplin N (2018) Confounding is not the only bias influencing associations of adiposity with cardiovascular disease. Eur Heart J 39:1521-1522. https://doi.org/10.1093/eurheartj/ehy133

42. Strandberg TE, Strandberg AY, Salomaa VV, Pitkala KH, Tilvis RS, Sirola J, Miettinen TA (2009) Explaining the obesity paradox: cardiovascular risk, weight change, and mortality during long-term follow-up in men. Eur Heart J 30:1720-1727. https://doi.org/10. 1093/eurheartj/ehp162

43. Suarez JI, Tarr RW, Selman WR (2006) Aneurysmal subarachnoid hemorrhage. N Engl J Med 354:387-396. https://doi.org/10.1056/ NEJMra052732

44. Tawk RG, Grewal SS, Heckman MG, Navarro R, Ferguson JL, Starke EL, Rawal B, Hanel R, Miller D, Wharen RE, Freeman WD (2015) Influence of body mass index and age on functional outcomes in patients with subarachnoid hemorrhage. Neurosurgery 76:136-141. https://doi.org/10.1227/NEU.0000000000000588

45. Wohlfahrt P, Lopez-Jimenez F, Krajcoviechova A, Jozifova M, Mayer O, Vanek J, Filipovsky J, Llano EM, Cifkova R (2015) The obesity paradox and survivors of ischemic stroke. J Stroke Cerebrovasc Dis 24:1443-1450. https://doi.org/10.1016/j. jstrokecerebrovasdis.2015.03.008

46. Yamada S, Koizumi A, Iso H, Wada Y, Watanabe Y, Date C, Yamamoto A, Kikuchi S, Inaba Y, Toyoshima H, Kondo T, Tamakoshi A, Japan Collaborative Cohort Study G (2003) Risk factors for fatal subarachnoid hemorrhage: the Japan Collaborative Cohort Study. Stroke 34:2781-2787. https://doi.org/ 10.1161/01.STR.0000103857.13812.9A

47. Zhao L, Du W, Zhao X, Liu L, Wang C, Wang Y, Wang A, Liu G, Wang Y, Xu Y (2014) Favorable functional recovery in overweight ischemic stroke survivors: findings from the China National Stroke Registry. J Stroke Cerebrovasc Dis 23:e201-e206. https://doi.org/ 10.1016/j.jstrokecerebrovasdis.2013.10.002

Publisher's note Springer Nature remains neutral with regard to jurisdictional claims in published maps and institutional affiliations. 